\title{
A GESTÃO DE QUALIDADE EM SAÚDE EM RELAÇÃO À SEGURANÇA DO PACIENTE: REVISÃO DE LITERATURA
}

HEALTH QUALITY MANAGEMENT IN RELATION TO PATIENT SAFETY: LITERATURE REVIEW

LA GESTIÓN DE CALIDAd EN SALUd EN RELACIÓN CON LA SEGURIDAd DEL PACIENTE: REVISIÓN DE LITERATURA

João Vitor Teixeira de Sousa ${ }^{1}$

Maria Sinara Farias 2

Palavras-chave:

Gestão em Saúde; Segurança do

Paciente; Revisão.

Keywords:

Health Management; Patient

Safety; Review.

Palabras clave:

Gestión de la Salud; Seguridad del Paciente; Revisión.

Submetido: 30/05/2019

Aprovado:

28/11/2019

Autor(a) para Correspondência:

João Vitor Teixeira de Sousa

R. Eubia Barroso, 4068

Cruzeiro - Itapipoca (CE)

62504-000

E-mail: joaovitorts11@hotmail.

\section{RESUMO}

Este artigo descreve, com base na literatura, ações realizadas pelos serviços de saúde em relação à segurança do paciente. Trata-se de revisão de literatura, com busca na Biblioteca Virtual em Saúde (BVS). Constatouse que as publicações datavam de $2010 \mathrm{em}$ diante e suas temáticas variam entre investigação da cultura de segurança, relação da segurança do paciente com a prevenção de eventos adversos e avaliação de incidentes, além da tradução de instrumento para avaliação da segurança do paciente. Os estudos ainda se mostram incipientes quanto às contribuições para a gestão, pois, apesar de relatarem a segurança como atributo de qualidade, não há pesquisas in loco que avaliem sua eficácia e efetividade. A análise nos leva à conclusão de que se mostra extremamente relevante discutir tanto a segurança do paciente quanto o gerenciamento dessa segurança, com vistas a contribuir para a melhoria da qualidade da assistência à saúde. Cabe destacar a necessidade da realização de novos estudos, pois a produção científica sobre esse tema ainda é escassa.
1. Especialista em Gestão de Saúde e Auditoria pelo Instituto Executivo de Formação (IEF). Enfermeiro no Hospital e Maternidade São Vicente de Paulo (São Camilo Itapipoca). E-mail: joaovitorts11@hotmail.com ORCID: https://orcid.org/0000-0002-8933-6779

2. Enfermeira. Mestre em Cuidados Clínicos em Enfermagem e Saúde pela Universidade Estadual do Ceará (UECE). E-mail: sinarafariasbc@gmail.com 0RCID: https://orcid.org/0000-0002-2695-502X 


\section{ABSTRACT}

This article describes, based on the literature, actions taken by health services regarding patient safety. This is a literature review, with search in the Virtual Health Library (VHL). It was found that the publications dated from 2010 onwards and their themes range from investigating safety culture, the relationship between patient safety and adverse event prevention, and the incident assessment, besides the translation of an instrument to assess patient safety. The studies still show to be incipient concerning the contributions to management, because, despite reporting safety as a good-quality attribute, there is no on-site research that evaluates its efficiency and effectiveness. The analysis leads us to the conclusion that it is extremely relevant to discuss both patient safety and patient safety management, with a view to contributing to improve health care quality. It is worth highlighting the need for further studies, as the scientific literature on this theme is still scarce.

\section{RESUMEN}

Este artículo describe, con base en la literatura, acciones tomadas por los servicios de salud con respecto a la seguridad del paciente. Esta es una revisión de literatura, con búsqueda en la Biblioteca Virtual en Salud (BVS). Se constató que las publicaciones datan de 2010 en adelante y sus temas van desde la investigación de la cultura de seguridad, la relación entre la seguridad del paciente y la prevención de eventos adversos, y la evaluación de incidentes, además de la traducción de un instrumento para evaluar la seguridad del paciente. Los estudios aún muestran ser incipientes con respecto a las contribuciones a la gestión, porque, a pesar de informaren que la seguridad es un atributo de buena calidad, no hay investigaciones in loco que evalúen su eficiencia y efectividad. El análisis nos lleva a la conclusión de que es extremadamente relevante discutir tanto la seguridad del paciente como la gestión de la seguridad del paciente, con el fin de contribuir a mejorar la calidad de la atención de salud. Vale la pena destacar la necesidad de más estudios, ya que la literatura científica acerca de este tema aún es escasa.

\section{INTRODUÇÃO}

Foi pensando na segurança do paciente, em termos da redução de riscos e agravos, que a Portaria n. 529, de $1^{\circ}$ de abril de 2013, foi instituída pelo Ministério da Saúde. Seu objetivo é contribuir para a qualificação do cuidado em todos os estabelecimentos de saúde do território nacional e tornar a abordagem tecnicista de segurança algo cultural ${ }^{1}$.

A cultura de segurança do paciente engloba 5 características:

1. Cultura na qual todos os trabalhadores, desde os profissionais envolvidos no cuidado até os gestores, assumem a responsabilidade por sua própria segurança e pela segurança de seus colegas e dos pacientes e seus familiares;

2. Cultura que prioriza a segurança acima de metas financeiras e operacionais;

3. Cultura que encoraja e recompensa a identificação, a notificação e a resolução dos problemas relacionados à segurança;

4. Cultura que, a partir da ocorrência de incidentes, promove o aprendizado organizacional; e

5. Cultura que proporciona recursos, estrutura e responsabilização para a manutenção efetiva da segurança².

0 termo cultura de segurança do paciente tem sido adotado sob a perspectiva de mudar a visão das pessoas sobre a forma de promover o cuidado seguro. É definido como a redução do risco de danos desnecessários associados à saúde, pois, considerando a complexidade dos procedimentos e tratamentos, o risco de danos significativos é real. 0 princípio dessa abordagem "cultural" é que os eventos adversos ou incidentes não são causados por má vontade das pessoas, mas por sistemas mal desenhados, e produzem resultados negativos perante a assistência prestada. Esse conceito cultural também faz alusão à introdução dos cuidados com segurança, de modo que façam parte do cotidiano dos serviços e que os erros não demandem soluções punitivas, mas agregadoras de conhecimento ${ }^{3}$.

Avaliar a cultura de segurança é importante para mensurar as condições organizacionais que possam facilitar a ocorrência de danos aos pacientes ${ }^{4}$. Tal avaliação é realizada para diagnosticar o nível de cultura de segurança entre os profissionais da saúde, os riscos de danos aos pacientes e as intervenções relacionadas aos erros, bem como o processo de notificação dos efeitos adversos. Avaliar a segurança do paciente requer envolvimento tanto dos profissionais quanto dos gestores no setor saúde ${ }^{5}$.

É comum que os enfermeiros, por vezes, assumam 
cargos na gestão de pessoas e na liderança de programas/sistemas de qualidade hospitalar. No atual cenário do setor saúde, esses profissionais militam pelo atendimento seguro, destacando 0 trabalho gerencial da enfermagem e demonstrando que esse é um dos alicerces das estratégias de segurança do paciente. Por isso, a articulação entre gerência e estratégias de segurança pode e deve ser investigada ${ }^{6}$.

Diante do exposto, investiga-se a atuação da equipe multiprofissional no que concerne à gestão e implantação de estratégias de segurança do paciente. Tal atuação pode subsidiar o planejamento de ações mais eficazes e efetivas, visando à superação de dificuldades no ambiente de trabalho e à implantação das estratégias de segurança do paciente.

0 estudo adotou algumas questões norteadoras:

- $\quad 0$ que existe na literatura sobre cultura de segurança do paciente?

- Quais são as ferramentas utilizadas para garantir uma boa adesão aos processos culturais de segurança?

- $\quad 0$ que essas publicações dizem aos gestores?

A relevância do estudo se sustenta na necessidade de conhecer o que existe na literatura sobre gestão em saúde e segurança do paciente e como esse processo de procedimentos seguros é implementado dentro dos serviços de saúde.

Os usuários dos serviços de saúde tendem a permanecer em estado de internação, expostos ao uso de inúmeras medicações, passíveis de interações medicamentosas e infecções, devido ao grande número de dispositivos invasivos em uso concomitante, principalmente na área hospitalar, e submetidos a polifarmácia, no âmbito da atenção primária à saúde (APS).

Este artigo descreve, com base na literatura, ações realizadas pelos serviços de saúde em relação à segurança do paciente.

\section{METODOLOGIA}

Trata-se de estudo do tipo revisão integrativa. Essa sintese possibilita agregar conhecimentos sobre determinado assunto, como a segurança do paciente, e aponta lacunas que necessitam ser preenchidas com a realização de novos estudos, que darão suporte à tomada de decisão do gestor quanto à temática em todos os ambientes, além de viabilizar uma síntese de múltiplos estudos publicados.

Seis fases compõem uma revisão integrativa.

\section{...necessidade \\ de conhecer o que \\ existe na literatura \\ sobre gestão \\ em saúde e segurança do paciente...}

Inicialmente, deve-se assimilar o tema e selecionar a hipótese ou questão de pesquisa. Em seguida, deve-se estabelecer os critérios de inclusão e exclusão de estudos. A terceira fase incide na demarcação das informações que serão extraídas dos estudos selecionados. Posteriormente, inicia-se a fase de avaliação dos estudos incluídos na revisão integrativa. A quinta fase consiste na interpretação dos resultados. $E$, por último, procede-se à redação e apresentação da revisão?.

As estratégias de busca na Biblioteca Virtual em Saúde (BVS) englobaram as seguintes bases de dados indexadas: a) Literatura Latino-Americana e do Caribe em Ciências da Saúde (LILACS); b) Banco de Dados em Enfermagem (BDENF); e c) Sistema Online de Busca e Análise de Literatura Médica (MedLine).

Adotou-se a seguinte questão de pesquisa:

- Quais ações estão sendo realizadas pelos serviços de saúde em relação à segurança do paciente?

A busca se deu a partir do cruzamento dos descritores "gestão em saúde" e "segurança do paciente", indexados no vocabulário controlado Descritores em Ciências da Saúde (DeCS), separados pelo operador booleano "AND". Foram listadas 4.777 obras, dentre elas: a) artigos; b) dissertações; e c) teses. Os critérios de inclusão foram: a) textos disponíveis na íntegra; e b) publicações em português (188 obras), tendo como assunto principal a gestão de segurança (43 obras). Já os critérios de exclusão foram: a) textos repetidos; e b) publicações que não se enquadrassem no propósito do estudo (dissertações de mestrado e teses de doutorado). Ao final, foram incluídos 14 artigos (2 indexados na BDENF; 6 na LILACS; 4 na MedLine; e 2 indexados em 2 bases de dados - BDENF e LILACS). Foram excluídos da análise os 2 artigos indexados em 2 bases, por consistirem em revisões de literatura, não suprindo o caráter primário de estudo. Não se delimitou um período de publicação.

Este estudo teve início em julho de 2018, com a 
elaboração do projeto de pesquisa, e findou-se em setembro de 2018, com a redação do relatório de pesquisa, sendo as buscas e a análise realizada em agosto do mesmo ano. A partir das informações obtidas foram elaborados quadros que ilustram os achados de pesquisa.

\section{RESULTADOS}

A segurança do paciente e a criação de uma cultura de segurança visam à redução de danos desnecessários e evitáveis aos pacientes durante a assistência à saúde. Essa discussão sobre segurança do paciente vem tomando grandes proporções desde a criação de políticas voltadas à temática, desenvolvidas inicialmente pela Organização Mundial da Saúde (OMS).

Portanto, trata-se de uma questão discutida mundialmente que tem gerado mudanças e instigado uma busca frequente e contínua pela melhoria da qualidade dos serviços de saúde, como mostra o Quadro 1 . 0 ano de publicação dos artigos confirma a relativa "juventude" dessa temática.

Quadro 1 - Artigos selecionados por ano de publicação. Sobral, 2018.

\begin{tabular}{|c|c|c|}
\hline & Título & Ano \\
\hline E1 & Cultura de segurança do paciente em hospital privado ${ }^{10}$ & 2018 \\
\hline E2 & $\begin{array}{l}\text { Cultura de segurança do paciente em três hospitais brasileiros com diferentes tipos de } \\
\text { gestã } 0^{5}\end{array}$ & 2018 \\
\hline E3 & $\begin{array}{l}\text { Fatores facilitadores na implantação das estratégias de segurança do paciente: estudo } \\
\text { descritivo-exploratório }{ }^{9}\end{array}$ & 2017 \\
\hline E4 & $\begin{array}{l}0 \text { enfermeiro: líder no gerenciamento de risco para prevenção e controle de infecções } \\
\text { em pacientes com câncer }{ }^{12}\end{array}$ & 2016 \\
\hline E5 & $\begin{array}{l}\text { Desenvolvimento e validação de indicadores de boas práticas de segurança do paciente: } \\
\text { Projeto ISEP-Brasil }{ }^{13}\end{array}$ & 2016 \\
\hline E6 & Adaptação transcultural e validação da escala de clima do trabalho em equipe ${ }^{14}$ & 2016 \\
\hline E7 & $\begin{array}{l}\text { Situação dos hospitais de referência para implantação/funcionamento do núcleo de } \\
\text { segurança do paciente }{ }^{15}\end{array}$ & 2016 \\
\hline E8 & $\begin{array}{l}\text { Estudo exploratório das iniciativas acerca da segurança do paciente em hospitais do Rio } \\
\text { de Janeiro }{ }^{16}\end{array}$ & 2014 \\
\hline E9 & Clima de segurança do paciente: percepção dos profissionais de enfermagem ${ }^{17}$ & 2012 \\
\hline E10 & $\begin{array}{l}\text { Estratégia educacional como contribuinte ao gerenciamento de risco hospitalar: estudo } \\
\text { quase-experimental } 20\end{array}$ & 2011 \\
\hline E11 & $\begin{array}{l}\text { Eventos adversos: análise de um instrumento de notificação utilizado no gerenciamento } \\
\text { de enfermagem }{ }^{18}\end{array}$ & 2010 \\
\hline E12 & $\begin{array}{l}\text { Fortalezas e ameaças em torno da segurança do paciente segundo a opinião dos } \\
\text { profissionais de enfermagem }{ }^{19}\end{array}$ & 2010 \\
\hline
\end{tabular}

Fonte: Elaborado pelos autores.

Como mostra o Quadro 2, dentre os 12 artigos analisados, temos: a) estudos descritivos (3); b) estudos transversais $(4) ; c$ ) estudo convergente assistencial (1); d) estudo metodológico (1); e) estudo avaliativo (1); f) estudo experimental (1); e g) relato de experiência (1).

0 Quadro 2 ilustra a qualidade das publicações, as tipologias de estudo e seus principais objetivos, facilitando a compreensão do objeto em análise. 
Quadro 2 - Distribuição dos dados de identificação dos artigos. Sobral, 2018.

\begin{tabular}{|c|c|c|c|c|c|c|}
\hline $\mathbf{N}$ & Ano & Periódico & Qualis & Autores & Objetivos & Tipo de estudo \\
\hline E1 & 2018 & $\begin{array}{l}\text { Revista de } \\
\text { Pesquisa: } \\
\text { Cuidado é } \\
\text { Fundamental } \\
\text { Online }\end{array}$ & B2 & $\begin{array}{c}\text { Golle, Ciotti, Herr, } \\
\text { Aozane, Schmidts, } \\
\text { Kolankiewicza }\end{array}$ & $\begin{array}{l}\text { Avaliar a percepção } \\
\text { dos profissionais de } \\
\text { enfermagem, atuantes } \\
\text { em um hospital } \\
\text { privado, acerca do } \\
\text { clima de segurança. }\end{array}$ & Estudo transversal \\
\hline E2 & 2018 & $\begin{array}{c}\text { Ciência \& } \\
\text { Saúde Coletiva }\end{array}$ & B1 & $\begin{array}{l}\text { Andrade, Lopes, } \\
\text { Souza Filho, Vieira } \\
\text { Junior, Farias, } \\
\text { Santos, et al. }\end{array}$ & $\begin{array}{l}\text { Avaliar a cultura de } \\
\text { segurança do paciente } \\
\text { e fatores associa- } \\
\text { dos em hospitais } \\
\text { brasileiros com } \\
\text { diferentes tipos de } \\
\text { gestão: federal, } \\
\text { estadual e privado. }\end{array}$ & $\begin{array}{c}\text { Estudo } \\
\text { observacional, } \\
\text { transversal, } \\
\text { com abordagem } \\
\text { analítica }\end{array}$ \\
\hline E3 & 2017 & $\begin{array}{l}\text { Online } \\
\text { Brazilian } \\
\text { Journal of } \\
\text { Nursing }\end{array}$ & B1 & $\begin{array}{l}\text { Oliveira, Reis, } \\
\text { Souza, Costa, } \\
\text { Valera, Matsuda }\end{array}$ & $\begin{array}{c}\text { Investigar, na } \\
\text { perspectiva de } \\
\text { enfermeiros gestores, } \\
\text { os meios/fatores que } \\
\text { facilitam o processo } \\
\text { de implantação } \\
\text { de estratégias } \\
\text { de segurança do } \\
\text { paciente. }\end{array}$ & $\begin{array}{l}\text { Estudo descritivo- } \\
\text { exploratório, } \\
\text { quantitativo }\end{array}$ \\
\hline E4 & 2016 & $\begin{array}{c}\text { Cogitare } \\
\text { Enfermagem }\end{array}$ & B1 & Sanhudo, Moreira & $\begin{array}{c}\text { Elaborar estratégias } \\
\text { de liderança em } \\
\text { enfermagem voltadas } \\
\text { à incorporação de } \\
\text { medidas de prevenção } \\
\text { e controle de } \\
\text { infecções relacionadas } \\
\text { à assistência à saúde } \\
\text { em pacientes com } \\
\text { câncer. }\end{array}$ & $\begin{array}{l}\text { Estudo } \\
\text { convergente } \\
\text { assistencial }\end{array}$ \\
\hline E5 & 2016 & $\begin{array}{l}\text { Cadernos de } \\
\text { Saúde Pública }\end{array}$ & $\mathrm{A} 2$ & $\begin{array}{c}\text { Gama, Saturno- } \\
\text { Hernández, } \\
\text { Ribeiro, Freitas, } \\
\text { Medeiros, Batista, } \\
\text { et al. }\end{array}$ & $\begin{array}{l}\text { Desenvolver e validar } \\
\text { indicadores de boas } \\
\text { práticas de segurança } \\
\text { do paciente para o } \\
\text { contexto brasileiro. }\end{array}$ & $\begin{array}{l}\text { Relato de } \\
\text { experiência }\end{array}$ \\
\hline E6 & 2016 & $\begin{array}{c}\text { Revista de } \\
\text { Saúde Pública }\end{array}$ & A2 & $\begin{array}{c}\text { Silva, Peduzzi, } \\
\text { Sangaleti, Silva, } \\
\text { Agreli, West, et al. }\end{array}$ & $\begin{array}{c}\text { Adaptar e validar a } \\
\text { escala "Team Climate } \\
\text { Inventory", de medida } \\
\text { do clima de trabalho } \\
\text { em equipe, para o } \\
\text { idioma português, no } \\
\text { contexto da atenção } \\
\text { primária à saúde no } \\
\text { Brasil. }\end{array}$ & $\begin{array}{l}\text { Estudo } \\
\text { metodológico }\end{array}$ \\
\hline E7 & 2016 & $\begin{array}{c}\text { Cogitare } \\
\text { Enfermagem }\end{array}$ & B1 & $\begin{array}{c}\text { Serra, Barbieri, } \\
\text { Cheade }\end{array}$ & $\begin{array}{l}\text { Conhecer a situação } \\
\text { dos hospitais de } \\
\text { referência quanto } \\
\text { ao uso de normas } \\
\text { e protocolos sobre } \\
\text { segurança do paciente } \\
\text { diante da implantação } \\
\text { da respectiva política } \\
\text { nacional. }\end{array}$ & Estudo transversal \\
\hline
\end{tabular}




\begin{tabular}{|c|c|c|c|c|c|c|}
\hline $\mathrm{N}$ & Ano & Periódico & Qualis & Autores & Objetivos & Tipo de estudo \\
\hline E8 & 2014 & $\begin{array}{c}\text { Revista } \\
\text { Enfermagem } \\
\text { UERJ }\end{array}$ & B1 & Souza, Silva & $\begin{array}{l}\text { Analisar, com base nas } \\
\text { informações obtidas } \\
\text { com os gerentes de } \\
\text { risco, as iniciativas } \\
\text { implementadas para } \\
\text { garantir a segurança do } \\
\text { paciente. }\end{array}$ & $\begin{array}{c}\text { Estudo exploratório- } \\
\text { descritivo }\end{array}$ \\
\hline E9 & 2012 & $\begin{array}{l}\text { Acta Paulista de } \\
\text { Enfermagem }\end{array}$ & $\mathrm{A} 2$ & $\begin{array}{c}\text { Rigobello, Carvalho, } \\
\text { Cassiani, Galon, } \\
\text { Capucho, Deus }\end{array}$ & $\begin{array}{c}\text { Avaliar a percepção } \\
\text { do clima de segurança } \\
\text { dos profissionais de } \\
\text { enfermagem atuantes } \\
\text { nas clínicas médicas e } \\
\text { cirúrgicas de um hospital } \\
\text { de ensino. }\end{array}$ & $\begin{array}{c}\text { Estudo transversal, } \\
\text { descritivo, } \\
\text { quantitativo }\end{array}$ \\
\hline E10 & 2011 & $\begin{array}{c}\text { Online Brazilian } \\
\text { Journal of } \\
\text { Nursing }\end{array}$ & B1 & $\begin{array}{l}\text { Silva, Barreiro Filho, } \\
\text { Santos, Nascimento }\end{array}$ & $\begin{array}{l}\text { Associar a estratégia } \\
\text { de sensibilização dos } \\
\text { profissionais associados } \\
\text { à Rede Sentinela ao } \\
\text { aumento de notificações } \\
\text { de eventos adversos. }\end{array}$ & $\begin{array}{l}\text { Estudo quase } \\
\text { experimental }\end{array}$ \\
\hline E11 & 2010 & $\begin{array}{c}\text { Revista da } \\
\text { Escola de } \\
\text { Enfermagem da } \\
\text { USP }\end{array}$ & $\mathrm{A} 2$ & Paiva, Paiva, Berti & $\begin{array}{c}\text { Analisar a utilização } \\
\text { desse instrumento } \\
\text { de comunicação e } \\
\text { identificar: frequência, } \\
\text { tipo, natureza e período } \\
\text { dos incidentes/eventos } \\
\text { adversos. }\end{array}$ & $\begin{array}{c}\text { Estudo retrospectivo } \\
\text { e descritivo }\end{array}$ \\
\hline E12 & 2010 & $\begin{array}{l}\text { Revista Latino- } \\
\text { Americana de } \\
\text { Enfermagem }\end{array}$ & A1 & $\begin{array}{l}\text { Ques, Montoro, } \\
\text { Gonzalez }\end{array}$ & $\begin{array}{c}\text { Identificar barreiras e } \\
\text { oportunidades que os } \\
\text { profissionais detectam } \\
\text { na prática clínica para } \\
\text { o desenvolvimento da } \\
\text { cultura de segurança dos } \\
\text { pacientes. }\end{array}$ & Pesquisa avaliativa \\
\hline
\end{tabular}

Fonte: Elaborado pelos autores.

Em relação à temática dos artigos, 7 faziam menção à investigação e avaliação da cultura de segurança do paciente; os demais versavam entre segurança do paciente associado a prevenção de eventos adversos e avaliação de incidentes, gerenciamento de risco, exceto 2 estudos, que tratavam da validação de indicadores de segurança do paciente, que auxiliam no gerenciamento de tal segurança.

\section{DISCUSSÃO}

A liderança no setor saúde é a chave para o desenvolvimento da segurança do paciente como cultura. Com o líder, os demais profissionais tendem a desenvolver métodos e estratégias de promoção do cuidado que sejam seguros para o paciente e para quem os aplica, além de sua alta qualidade. 0 gerenciamento dos setores quanto à segurança do paciente traz maior seguridade e promove um ambiente adequado, longe da cultura da não notificação e da punição severa aos erros, já hegemonizada e sustentada em várias instituições de saúde ${ }^{8}$.

Fatores como carga de trabalho alta, equipe reduzida, problemas de comunicação entre os membros da equipe, falta de insumos necessários à realização de procedimentos e falta de apoio gerencial constituem fatores desencadeantes de uma assistência insegura e de baixa qualidade. Para uma cultura de segurança do paciente eficaz e realmente levada a sério, mostra-se indispensável o trabalho em equipe; para que isso ocorra, faz-se necessário o apoio de quem gerencia o setor, com um olhar voltado ao entendimento de que a segurança leva à excelência ${ }^{8}$. 
A liderança dentro da equipe engloba a capacidade de dirigir, coordenar e avaliar a performance dos membros que a compõem, além de atribuir tarefas e promover educação continuada, a fim de motivar os integrantes a organizarem e estabelecerem uma atmosfera positiva dentro do local de trabalho ${ }^{9}$.

Nesse contexto, vale destacar que os serviços de saúde contemporâneos levam os profissionais a realizar uma grande quantidade de atividades, muitas vezes sob pressões de tempo e frequente escassez de recursos. Ao contrário das falhas ativas, que muitas vezes são difíceis de prever, as condições latentes podem ser identificadas e corrigidas de modo preventivo quando há gerenciamento de risco proativo e não reativo. Nesse caso, é essencial migrar de uma cultura punitiva para métodos de aprendizagem que levam à abertura de comunicação, possibilitando que os profissionais falem de modo aberto sobre os erros que podem afetar significativamente o paciente ${ }^{8,10}$.

Levando em consideração o parágrafo anterior, um estudo transversal publicado em 2018 evidenciou distância entre a gerência de enfermagem e a gerência hospitalar em relação aos trabalhadores in loco. Isso mostra a necessidade de ampliar o olhar da gestão, com o intuito de melhorar os indicadores de segurança do paciente e, por consequência, obter mudanças culturais que podem vir a favorecer a assistência à saúde, tornando-a mais segura e fortalecendo o vínculo entre os profissionais ${ }^{10}$.

Em outro estudo, publicado em 2018, foram pesquisados 3 tipos diferentes de gerência em hospitais: a) federais; b) estaduais; e c) privados. Evidenciou-se que as áreas prioritárias para ações que visem à melhoria da cultura de segurança devem enfocar as dimensões "Frequência de eventos notificados", "Percepção de segurança", "Feedback e comunicação sobre os erros", "Resposta não punitiva para erros", "Dimensionamento de pessoal", "Trabalho em equipe entre unidades" e "Problemas em mudanças de turno e transições entre unidades/serviços". Essas foram as áreas em que ocorreram os menores percentuais de respostas positivas quando se analisou 0 agregado de hospitais, nas 3 esferas estudadas ${ }^{5}$.

Em um estudo semelhante, que investigou a atuação do enfermeiro como gestor em quatro hospitais distintos, foi demonstrado e afirmado pelos sujeitos de pesquisa que o apoio da alta gestão hospitalar é um facilitador da implantação de estratégias de cuidado seguro. Isso é importante porque, quando a direção/

\section{...o apoio \\ da alta gestão \\ hospitalar é um \\ facilitador da \\ implantação de \\ estratégias de cuidado \\ seguro...}

gestão organizacional demonstra compromisso com a qualidade da assistência prestada, a implementação de intervenções passa a ser mais fácil. Nesse cenário da gestão hospitalar, sabe-se que, muitos hospitais ainda são geridos de modo arcaico, sendo os modelos de gestão, centralizados, aquilo que dificulta a comunicação e, consequentemente, a efetivação de melhorias e mudanças nos processos de atendimento. Com isso, deve-se englobar as necessidades organizacionais, a segurança do paciente como um bem desejável a ser gerenciado ${ }^{9}$.

Dentro do contexto de segurança, a equipe de enfermagem é responsável por contribuir com métodos educacionais e reeducacionais, para que se desenvolva o senso de cultura, como a identificação erros, que não levem a punições, mas ao amparo ao refinamento da segurança do paciente. A enfermagem desenvolve um papel importante na oferta de assistência segura e eficaz, sendo que se encontra 24 horas por dia com o paciente, tornando-se responsável por identificar e registrar os eventos adversos, expondo aos demais membros e profissionais da equipe a importância de desenvolver um ambiente seguro para todos, sejam profissionais ou pacientes ${ }^{11}$.

Em uma pesquisa realizada com enfermeiros gerentes de unidades oncológicas, verificou-se que as estratégias de liderança devem ser implementadas em todos os setores do hospital, porém, existem os locais prioritários para a implementação dessa liderança/gerência. Eles trazem que muitos enfermeiros ficam sobrecarregados ao assumir outros setores concomitantemente, por isso, deixam de acompanhar o trabalho em equipe da maneira correta ou como gostariam. Essa situação aponta uma reflexão sobre a relação do dimensionamento de pessoal com a demanda de serviços dentro do setor. $E$, novamente, engloba a gestão, onde se mostra necessário um olhar voltado ao preenchimento ou à criação de vagas, que são supridas de forma 
sobrecarregada pelos profissionais já existentes, sem que tenham acréscimos de remuneração por tal feito ${ }^{12}$

Métodos já vêm sendo criados com a intenção de qualificar e proporcionar suporte ao gerenciamento da segurança do paciente. Indicadores já validados contribuem para a gestão da segurança do paciente, assim como instrumentos validados para avaliação da cultura de segurança do paciente, e apresentam validade, confiabilidade, viabilidade e potencial utilidade para o enfrentamento de riscos assistenciais. Com o novo conceito de segurança do paciente, mencionado como algo cultural, é de grande importância a presença de medidas de diminuição do risco de dano, prevenindo-o e usando abordagens inovadoras quanto à ocorrência de um possivel incidente. Indicadores e instrumentos de avaliação têm potencial para ser incorporados às políticas públicas, porém, ainda são escassos, o que dificulta o monitoramento, com a perspectiva de avaliar as práticas seguras ${ }^{13-14}$.

Em um estudo realizado em 2016, foi levada em questão a ótica organizacional, bem como o desenvolvimento e a implementação de protocolos que afiram medidas tomadas em relação à redução de gastos excessivos que, em caso contrário, poderiam constituir recursos investidos em setores como infraestrutura, apoio às compras hospitalares e reposição de almoxarifado, melhorando a distribuição de insumos dentro dos serviços e garantindo uma melhora na eficiência institucional como um todo. A segurança do paciente deve ser vista como um ecossistema, onde os serviços de apoio, a infraestrutura e as tecnologias, devem apoiar deliberadamente os processos de trabalho, contribuindo para 0 melhor gerenciamento da assistência e, consequentemente, uma melhora da qualidade do serviço. Fica posto neste estudo que os hospitais que almejam chegar a zero ocorrência de eventos adversos devem abraçar políticas de integração, que cerquem toda o corpo hospitalar, trabalhando efetivamente a mudança da cultura - eis o papel primordial da gestão, implantar tal cultura de modo eficaz e sem comprometer a integridade dos profissionais ${ }^{15}$.

As iniciativas tomadas em alguns hospitais do Rio de Janeiro incluem a identificação do paciente e a assistência limpa e constatou-se que os gerentes hospitalares desenvolvem ações intrainstitucionais, quais sejam: a) cursos para suporte de educação continuada; b) orientações da comissão de controle

\section{A segurança do \\ paciente deve ser \\ vista como um \\ ecossistema...}

de infecções hospitalares; c) elaboração de protocolos; d) folders; e) manuais; e f) e check-list de cirurgia segura. Os gerentes informaram, ainda, que adotam o método de educação continuada como uma estratégia de disseminação de conhecimento, para que seja fortalecida a segurança do paciente de modo contextualizado no ambiente hospitalar e, ainda mais importante, que essa segurança seja cultural ${ }^{16}$.

Contrapondo-se ao exposto, realizou-se uma pesquisa em 2016 com enfermeiros de São Paulo sobre a percepção da segurança do paciente, porém, constatou-se baixo nível de escores de respostas positivas em relação à percepção desses profissionais quanto à gerência hospitalar e estes últimos, segundo os enfermeiros, mantêm-se distantes da equipe de enfermagem, sendo hierárquica a debilidade do diálogo. Verificou-se que as falhas na segurança do paciente, neste estudo, resultam em sua grande maioria em ações punitivas para o profissional, o que torna o assunto de difícil discussão, fazendo com que a segurança como cultura fique em segundo plano, o que não traz as melhorias necessárias à saúde e à redução de riscos ao paciente ${ }^{17}$.

Vale lembrar que as ações de educação em saúde e educação continuada se mantenham no topo das ações primordiais para a organização e no início de uma abordagem cultural em relação à segurança do paciente. Cartazes, folhetos e palestras são fatores contribuintes para o aumento do número de notificações de eventos adversos, aumentando a possibilidade de maior controle sobre as ocorrências do serviço pelo gestor e possibilitando a correção desses erros de modo sábio, sem a necessidade de punições individuais, tendo como base treinamentos e exposições coletivas ${ }^{18-19}$.

Afinal, introduzir práticas de qualificação dos serviços, com ênfase em uma cultura organizacional, não é algo rápido; trata-se de um processo lento, que requer apoio de vários setores (como saúde e educação), onde possa haver maior incentivo do Sistema Único de Saúde (SUS) à formação para 
a saúde e à visão cultural dos processos, seja em termos de qualidade, segurança ou humanização.

\section{CONCLUSÃO}

Este estudo apresenta evidências científicas sobre a temática segurança do paciente, indo da abordagem de conteúdos de natureza avaliativa até medidas preventivas e aspectos culturais pertinentes.

Também se observou a ausência de produções científicas voltadas integralmente à gerência de risco ao paciente, tendo como ênfase a abordagem cultural, o que pode ser apontado como uma limitação deste estudo.

Nesse contexto, muitos espaços vazios de conhecimentos perpassam o estudo do tema, como poucas informações sobre cultura de segurança entre os profissionais, visto que o termo é relativamente novo, fazendo com que haja, ainda, escassez de estratégias de abordagem e sensibilização diante dos instrumentos de investigação de fácil acesso, impossibilitando a alta gestão de tomar decisões baseadas nessas evidências, tendo em vista a baixa quantidade de estudos avaliativos com uma avaliação mais qualificada e resolutiva.

Almeja-se que este estudo estimule a realização de novas pesquisas sobre a cultura de segurança do paciente e o gerenciamento dessa segurança, para que possam ser criadas novas estratégias de sensibilização e divulgação da temática, promovendo práticas mais eficientes entre os profissionais em todos os âmbitos do setor saúde e a consequente qualificação dos serviços.

\section{CONTRIBUIÇÃO DOS AUTORES}

João Vitor Teixeira de Sousa contribuiu com a realização da pesquisa, o delineamento do estudo e a redação do manuscrito. Maria Sinara Farias contribuiu com a redação e a revisão crítica do manuscrito.

\section{REFERÊNCIAS}

1. Brasil. Norma Operacional Básica do Sistema Único de Saúde/NOB-SUS 96. Brasília (DF): Ministério da Saúde; 1997.

2. Brasil. Portaria MS/GM n. 529, de $1^{\circ}$ de abril de 2013. Brasília (DF): Ministério da Saúde; 2013.
3. Leape LL. Errors in medicine. Clin Chim Acta. $2009 ;(404): 2-5$.

4. Brasil. Implantação do Núcleo de Segurança do Paciente em Serviços de Saúde. Brasília (DF): Anvisa; 2014. (Série Segurança do Paciente e Qualidade em Serviços de Saúde).

5. Andrade LEL, Lopes JM, Souza Filho MCM, Vieira Junior RF, Farias LC, Santos CCM, et al. Cultura de segurança do paciente em três hospitais brasileiros com diferentes tipos de gestão. Ciênc Saúde Colet. 2018;23(1):161-72.

6. Oliveira AM, Rodrigues VAV, Passerini JP, Pedreiro PBZ, Minto BA. Queixas técnicas e reações adversas a medicamentos notificadas em um hospital regional no Brasil: um estudo transversal. ABCS Health Sci [serial on the internet]. 2018 [cited 2019 Dec $16] ; 43(1): 25-9$. Available from: file:///D:/10152602-1-PB.pdf

7. Mendes KDS, Silveira RCCP, Galvão CM. Revisão integrativa: método de pesquisa para a incorporação de evidências na saúde e na enfermagem. Texto \& Contexto Enferm [serial on the internet]. 2008 [cited 2019 Dec 16];17(4):758-64. Available from: http://www.scielo.br/pdf/tce/v17n4/18.pdf

8. Lemos GC, Azevedo C, Bernardes MFVC, Ribeiro HCTC, Menezes AC, Mata LRF. A cultura de segurança do paciente no âmbito da enfermagem: reflexão teórica. Revista de Enfermagem do Centro-0este Mineiro [serial on the internet]. 2018 [cited 2019 Dec 16];(8):2600. Available from: http://www.seer.ufs]. edu.br/index.php/recom/article/view/2600/1880

9. Oliveira JLC, Reis GAX, Souza VS, Costa MAR, Valera IMA, Matsuda LM. Fatores facilitadores na implantação das estratégias de segurança do paciente: estudo descritivo-exploratório. Online Braz J Nurs [serial on the internet]. 2017 [cited 2019 Dec 16];16(2):108-18. Available from: http:// objnursing.uff.br/index.php/nursing/article/ viewFile/5515/pdf 2

10. Golle L, Ciotti D, Herr GEG, Aozane F, Schmidts CR, Kolankiewicza AC. Cultura de segurança do paciente em hospital privado. Rev Pesqui (Univ Fed Estado Rio J, Online) [serial on the internet]. 2018 [cited 2019 Dec 16];10(1):85-9. Available from: file:///D:/598034289-3-PB.pdf

11. Miranda AP, Carvalho AKO, Lopes AA, Oliveira VRC, Carvalho PMG, Carvalho HEF. Contribuição da enfermagem à segurança do paciente: revisão integrativa. Sanare (Sobral, Online) [serial on the internet]. 2017 [cited 2019 Dec 16];16(1):109-17. Available from: https://sanare.emnuvens.com.br/ sanare/article/view/1101/612 
12. Sanhudo NF, Moreira MC. 0 enfermeiro: líder no gerenciamento de risco para prevenção e controle de infecções em pacientes com câncer. Cogitare Enferm [serial on the internet]. 2016 [cited 2019 Dec 16];21(3):1-9. Available from: https://revistas. ufpr.br/cogitare/article/view/45590

13. Gama ZAS, Saturno-Hernández PJ, Ribeiro DNC, Freitas MR, Medeiros PJ, Batista AM, et al. Desenvolvimento e validação de indicadores de boas práticas de segurança do paciente: Projeto ISEPBrasil. Cad Saúde Pública [serial on the internet]. 2016 [cited 2019 Dec 16];32(9):e00026215. Available from: http://www.scielo.br/pdf/csp/v32n9/16784464-csp-32-09-e00026215.pdf

14. Silva MC, Peduzzi M, Sangaleti CT, Silva D, Agreli HF, West MA, et al. Adaptação transcultural e validação da escala de clima do trabalho em equipe. Rev Saúde Pública [serial on the internet]. 2016 [cited 2019 Dec 16];22(50):1-10. Available from: https://www.scielosp.org/article/ssm/content/ raw/?resource ssm path $=/ \mathrm{media} /$ assets $/ \mathrm{rsp} / \mathrm{v} 50 /$ pt 0034-8910-rsp-S1518-87872016050006484.pdf

15. Serra JN, Barbieri AR, Cheade MFM. Situação dos hospitais de referência para implantação/ funcionamento do núcleo de segurança do paciente. Cogitare Enferm [serial on the internet]. 2016 [cited 2019 Dec 16];(21):1-9. Available from: https:// revistas.ufpr.br/cogitare/article/view/45925/pdf

16. Souza RFF, Silva LD. Estudo exploratório das iniciativas acerca da segurança do paciente em hospitais do Rio de Janeiro. Rev Enferm UERJ [serial on the internet]. 2014 [cited 2019 Dec 16];22(1):228. Available from: https://www.e-publicacoes. uerj.br/index.php/enfermagemuerj/article/ view/11399/8972

17. Rigobello MCG, Carvalho REFL, Cassiani SHB, Galon T, Capucho HC, Deus NN. Clima de segurança do paciente: percepção dos profissionais de enfermagem. Acta Paul Enferm [serial on the internet]. 2012 [cited 2019 Dec 16];25(5):728-35. Available from: http://www.scielo.br/pdf/ape/v25n5/13.pdf

18. Paiva MCMS, Paiva SAR, Berti HW. Eventos adversos: análise de um instrumento de notificação utilizado no gerenciamento de enfermagem. Rev Esc Enferm USP [serial on the internet]. 2010 [cited 2019 Dec 16];44(2):287-94. Available from: http:// www.scielo.br/pdf/reeusp/v44n2/07.pdf

19. Ques AAM, Montoro CH, Gonzalez MG. Fortalezas e ameaças em torno da segurança do paciente segundo a opinião dos profissionais de enfermagem. Rev Latinoam Enferm [serial on the internet]. 2010 [cited 2019 Dec 16];18(3). Available from: http:// www.scielo.br/pdf/rlae/v18n3/pt 07.pdf

20. Silva RFA, Barreiro Filho RD, Santos M, Nascimento MAL. Estratégia educacional como contribuinte ao gerenciamento de risco hospitalar: estudo quaseexperimental. Online Braz J Nurs. 2011;10(1):1-8. Available from: http://www.objnursing.uff.br/index. $\mathrm{php/nursing/article/view/j.1676-4285.2011.3221.1/}$ $\underline{\mathrm{html}}$
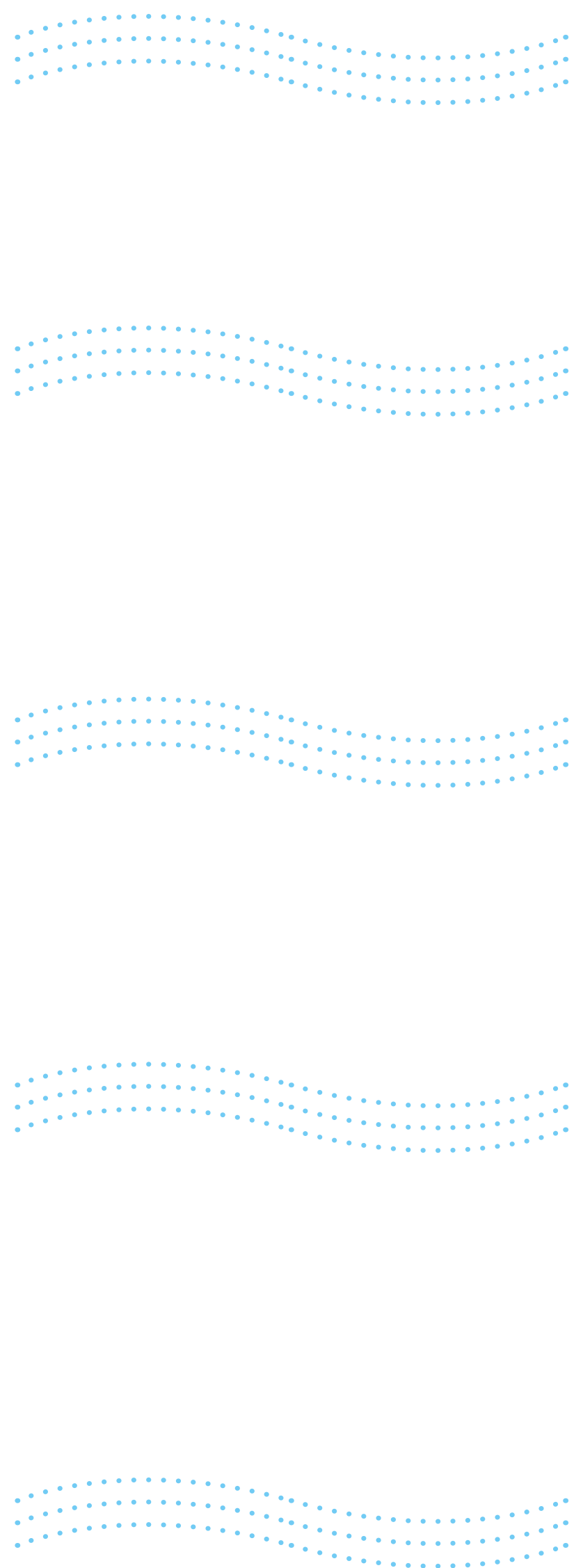

SANARE (Sobral, Online). 2019 Jul-Dec;18(2):96-105 - 105 